\title{
The Ejection Fraction Returns to Hyde Park Session's Speakers' Corner
}

\author{
Wolney de Andrade Martins ${ }^{1,2}$ and Antonio José Lagoeiro Jorge ${ }^{1}$ \\ Universidade Federal Fluminense (UFF), ${ }^{1}$ Niterói, RJ - Brazil \\ Complexo Hospitalar de Niterói (CHN), ${ }^{2}$ Niterói, RJ - Brazil
}

The ejection fraction returns to Hyde Park Session's Speakers' Corner

About two decades ago, the Heart Failure Society of America created the Hyde Park Session at its annual meeting. ${ }^{1}$ The analogy with the free and innovative proposals that took place in the historic London park were transposed to that scientific event. One of the first proposals made there was the extinction of the left ventricular ejection fraction (LVEF) as if it was an absolutist tyrant determining the life of his/her subjects. The proposition took no breath. The overwhelming majority of heart failure (HF) trials used LVEF as an inclusion criterion. The cutoff points varied. Magical numbers had little pathophysiological or clinical foundation. LVEF was measured by noninvasive methods, especially echocardiography, with high intraand inter-examiner variability, dependent on preload and afterload changes. ${ }^{2}$

Later, in 2001, a clinical entity was recognized, where acute pulmonary edema of cardiogenic cause occurred despite LVEF at levels above $50 \% .^{3}$ The following years saw incredulous initial acceptance until the epidemiological and clinical characterization of what we today call "heart failure with preserved ejection fraction (HFpEF)." From rare, it became frequent, especially in primary care. Considered benign, the prognosis became almost as reserved as heart failure with reduced ejection fraction (HFrEF). There were different proposals for diagnosis, prognostic scores, but such evolution in the knowledge of HFpEF resulted in a frustrating succession of negative therapeutic trials.

\section{Keywords}

Heart Failure/physiopathology; Stroke Volume/ physiology; Ventricular Dysfunction; Prognosis.
In 2013, ACCF/AHA ${ }^{4}$ re-stratified LVEF levels and created "borderline heart failure," which was in fact settled by the 2016 European guideline ${ }^{5}$ under the "mid-range" nomenclature. Something was created that was not known in depth. The race for the demographic, clinical and prognostic characterization of the new entity began. What is heart failure with mid-range ejection fraction (HFmrEF)? Which direction of travel? HFrEF in reverse remodeling under optimal treatment? HFpEF following natural history with progressive necrosis, fibrosis and dilation vis-à-vis lack of treatment? An early manifestation of the disease? Would these different phenotypes grouped together by LVEF strata have the same clinical behavior? Tsuji et al., ${ }^{6}$ showed that the clinical characteristics of HFmrEF are intermediate between HFpEF and HFrEF and that HFmrEF has a dynamic transition to HFpEF or HFrEF, especially within a year, then suggesting that HFmrEF would represent a transition phenotype or an overlap zone between HFpEF and HFrEF instead of an independent heart failure entity. Currently, it is known that there are many HF phenotypes besides the simplification of LVEF strata.

There are few studies addressing the HFpEF and HFmrEF strata, either due to the relative novelty of HFrEF or the need for inclusion in clinical trials of lower LVEF patients, where the expectation of mortality and major events would increase the statistical power of the study. ${ }^{2}$ Currently, there is a tendency for higher valuation of studies that include higher LVEF.

The paper published by Cavalcanti et al.,7 in the International Journal of Cardiovascular Sciences draws a picture of 493 patients admitted for decompensated HF in the northeast region of Brazil over a 10-year period. Then, it compares the three strata defined by the ESC: reduced, mid-range and preserved. If we break free from the dictatorship of the $p$-value, we can see from the results 
of the study that patients who presented themselves as HFmrEF now resemble each other's extremes. They resemble HFrEF in NYHA functional class, etiologies, chronic kidney disease, valvular heart disease, alcoholism and smoking, prevalence of atrial fibrillation, mitral and tricuspid regurgitation, and B-type natriuretic peptide levels. On the other hand, they resemble HFpEF as to age, presence of hypertension and diabetes mellitus, anemia and right ventricular remodeling. Mortality appears to be closer to HFpEF and 30-day readmission to HFrEF. These last two facts lose magnitude, since all numbers are alarming.

Cavalcanti et al., ${ }^{7}$ presented a frequency of $26 \%$ of patients with HFmrEF, as well as other characteristics consistent with those recently described in the literature. ${ }^{6,8,9}$ It is very important that we know this characterization, because the answer on how to treat depends on it.
The HFrEF prescription, very well grounded in large clinical trials or the therapeutic uncertainties of HFpEF. Cavalcanti et al., ${ }^{7}$ show data that should reflect the reality of a tertiary or quaternary referral hospital in a population with a higher socioeconomic level. We should contextualize this. It may not be reproduced in primary care or in public institutions.

The still very high 30-day mortality and readmission rates presented by Cavalcanti et al., ${ }^{7}$ make it mandatory that we improve the approach to HF as a whole, independent of LVEF. It is important to have the demographic and clinical portrait of HFpEF and HFmrEF. The paper presented by Cavalcanti et al., ${ }^{7}$ is an important contribution to one of the obscure areas of HF. We do hope that the debate at the Hyde Park session should be based on evidence such as the one presented by Cavalcanti et al. ${ }^{7}$

\section{References}

1. Heart Failure Society of America.(HFSA). [Internet]. [Cited in 2019 Oct 11]. Available from: https://meeting.hfsa.org/past-meetings/

2. Pfeffer MA, Shah AM, Borlaug BA. Heart failure with preserved ejection fraction in perspective. Circ Res. 2019;124(11):1598-617.

3. Gandhi SK, Powers JC, Nomeir AM, Fowle K, Kitzman DW, Rankin $\mathrm{KM}$, et al. The pathogenesis of acute pulmonary edema associated with hypertension. N Engl J Med. 2001;344(1):17-22.

4. Yancy CW, Jessup M, Bozkurt B, Butler J, Casey DE Jr, Drazner MH, et al. 2013 ACCF/AHA guideline for the management of heart failure: a report of the American College of Cardiology Foundation/American Heart Association Task Force on Practice Guidelines. J Am Coll Cardiol 2013;62(16):e147-239.

5. Ponikowski P, Voors AA, Anker SD, Bueno H, Cleland JGF, Coats AJS., et al. 2016 ESC guidelines for the diagnosis and treatment of acute and chronic heart failure: the Task Force for the diagnosis and treatment of acute and chronic heart failure of the European Society of Cardiology (ESC) developed with the special contribution of the Heart Failure Association (HFA) of the ESC. Eur Heart J. 2016;37(36):2129-200.

6. Tsuji K, Sakata Y, Nochioka K, Miura M, Yamauchi T, Onose T., et al. Characterization of heart failure patients with midrange left ventricular ejection fraction-a report from the CHART-2 study. Eur J Heart Fail. 2017;19(10):1258-69.

7. Cavalcanti CP, Sarteschi C, Gomes GES, Medeiros CA, Pimentel JHM, Lafayette AR, et al. Decompensated heart failure with mid-range ejection fraction: epidemiology and inhospital mortality risk factors. Int J Cardiovasc Sci. 2020;33(1):45-54.

8. Kapoor JR, Kapoor R, Ju C, Heidenreich PA, Eapen ZJ, Hernandez AF, et al. Precipitating clinical factors, heart failure characterization, and outcomes in patients hospitalized with heart failure with reduced, borderline, and preserved ejection fraction. J Am Coll Cardiol HF. 2016;4(6):464-72.

9. Lauritsen J, Gustafsson F, Abdulla J. Characteristics and long-term prognosis of patients with heart failure and mid-range ejection fraction compared with reduced and preserved ejection fraction: A systematic review and meta-analysis. ESC Heart Fail. 2018;5(4):685-94. 